Revista Eletrônica de Ciência Administrativa (RECADM) - ISSN 1677-7387

Faculdade Cenecista de Campo Largo - Coordenação do Curso de Administração

v. 4, n. 1, maio/2005 - http://revistas.facecla.com.br/index.php/recadm/

\title{
LA ESTRUCTURA FINANCIERA DE LAS EMPRESAS. UN ANÁLISIS DESCRIPTIVO ${ }^{1}$
}

Dr. Félix Jiménez Naharro, fjimenez@us.es, Universidad de Sevilla

Dra. María José Palacín Sánchez, palacin@us.es, Universidad de Sevilla

\section{RESUMEN}

El estudio de la estructura financiera de las empresas sigue siendo uno de los temas financieros que más interés suscita, entre otros motivos y según Myers (2001), porque no existe una teoría universal sobre la estructura financiera y, es mas, no hay razones para esperarla. Partiendo de esta situación nos planteamos analizar los efectos de la dimensión empresarial y de pertenencia a un sector, dos factores tradicionalmente aceptados por la literatura financiera como determinantes de la estructura de capital, sobre la estructura financiera de las empresas pertenecientes a la Comunidad Autónoma Andaluza. Para ello hemos utilizado una muestra de 6.338 empresas andaluzas en el periodo 2001. Los resultados reflejan como las empresas andaluzas están muy endeudadas, siendo las empresas de reducida dimensión, y las que pertenecen a los sectores de la construcción y servicios las más apalancadas.

PALABRAS CLAVE: estructura de capital, sector de actividad, tamaño.

\section{RESUMO}

Este papel analisa a relevância de dois fatores chaves em determinar a estrutura de capitais das companhias: dimensao e sector de actividade das empresas. INossa análise foi realizada em uma mostra de 6.338 empresas andalusian para o período 2001. ๆOs resultados de nosso trabalho mostram que as compañías andalusian são altamente endividadas. ๆAlém das firmas pequenas são mais nível do endividamento do que as médias e grandes empresas. TOs resultados indicam também que as companhias que pertencem aos setores de serviços da construção e do negócio têm as relações as mais elevadas do débito. ๆ

\footnotetext{
${ }^{1}$ Este trabajo ha sido realizado dentro del marco del Grupo de Investigación: "Nuevos Desarrollos en Finanzas" (SEJ 381), y financiado con los fondos procedentes de la Ayuda a la Consolidación de Grupos de Investigación dentro del III PAI.
} 
Revista Eletrônica de Ciência Administrativa (RECADM) - ISSN 1677-7387

Faculdade Cenecista de Campo Largo - Coordenação do Curso de Administração

v. 4, n. 1, maio/2005 - http://revistas.facecla.com.br/index.php/recadm/

PALAVRAS-CHAVES: dimensao da empresa, estrutura de capitais, classificação da indústria,

\section{ABSTRAC}

This paper analyse the relevance of two key factors in determining the capital structure of companies: firm size and industry classification. Our analysis has been carried out on 6.338 andalusian companies for 2001 period. The results of our study show that andalusian companies are highly leveraged. Besides small firms are more level of debt than medium and large firms. The results also indicate that the companies that belong to construction and business services sectors have the highest debt ratios.

KEY WORDS: capital structure, industry classification, size firm.

\section{INTRODUCCIÓN}

Nuestro trabajo se encuadra dentro de una de las áreas de investigación más compleja en finanzas como es la que tiene por objeto el estudio de la estructura financiera. Son muchos los estudios teóricos y empíricos que se han realizado para intentar explicar la estructura financiera, esto es, la combinación de recursos propios y ajenos utilizados por las empresas para financiar sus inversiones. A estas alturas del nuevo siglo y siguiendo a Myers (2001), podemos afirmar que no existe una teoría universal sobre la estructura financiera y, es mas, no hay razones para esperarla. Sin embargo sí existen varias teorías parciales de gran utilidad.

Partiendo de esta situación nos planteamos analizar los efectos de la dimensión empresarial y de pertenencia a un sector sobre la estructura financiera, tomando como base del estudio las empresas pertenecientes a la Comunidad Autónoma Andaluza. Es decir pretendemos testar si existen o no diferencias significativas en la estructura financiera según la clase de tamaño y de sector a que pertenecen las empresas, y en caso afirmativo determinar la dirección de tales diferencias. Este tipo de estudio es especialmente interesante para evaluar las políticas públicas orientadas a las PYMES, que se han desarrollado en esta 
Revista Eletrônica de Ciência Administrativa (RECADM) - ISSN 1677-7387

Faculdade Cenecista de Campo Largo - Coordenação do Curso de Administração

v. 4, n. 1, maio/2005 - http://revistas.facecla.com.br/index.php/recadm/

comunidad, puesto que estas políticas se articulan agrupando a las empresas por clases de tamaño, e incluso por sector de interés.

El artículo se organiza de la siguiente manera. Primero se exponen las diferentes teorías financieras que han ido surgiendo a lo largo del tiempo para explicar la estructura financiera de la empresa. En segundo lugar, se describe la muestra de empresas y las variables a estudiar. En tercer lugar se presenta la metodología estadística empleada para realizar nuestro estudio y se valoran los resultados alcanzados, y por último se establecen las conclusiones.

Este trabajo pretende sentar las bases para la realización, en una fase posterior, de un estudio estadístico más completo y riguroso que persigue testar la significación de las diferencias en estructura financiera entre las empresas andaluzas identificadas en el mismo.

\section{TEORÍAS FINANCIERAS SOBRE LA ESTRUCTURA FINANCIERA}

No podemos comenzar esta revisión de las teorías sobre la estructura financiera sin citar el trabajo seminal de Modigliani y Miller (1958), en el que demostraban que suponiendo que los mercados financieros eran perfectos, el cómo la empresa se financiase no afectaba al valor de mercado de la corporación, porque lo que de verdad determinaba dicho valor eran las inversiones, es decir el tamaño y el riesgo de los flujos de caja que generaban los activos de la empresa. Aunque a dicha tesis de la irrelevancia de la estructura financiera y en las condiciones que ellos la definieron, no se le puedan poner reparos, no es menos cierto que dicha teoría resulta absolutamente insuficiente para explicar la elección de la estructura financiera de las empresas actuales en los modernos, pero imperfectos, mercados financieros.

Una de las corrientes que más fuerza ha tenido para explicar la estructura financiera, en realidad en esa línea se movieron ya Modigliani y Miller, es la llamada Teoría del Óptimo Financiero (Trade-off Theory). Esta teoría sugiere la existencia de una estructura de capital óptima para cada empresa, es decir, existe una combinación de recursos propios y deuda que maximiza el valor de mercado de la empresa y minimiza el coste medio de los recursos. Ese óptimo de deuda se alcanza donde se equilibren los beneficios y costes asociados a una unidad adicional de 
Revista Eletrônica de Ciência Administrativa (RECADM) - ISSN 1677-7387

Faculdade Cenecista de Campo Largo - Coordenação do Curso de Administração

v. 4, n. 1, maio/2005 - http://revistas.facecla.com.br/index.php/recadm/

deuda. Entre las ventajas asociadas al endeudamiento se encuentran: la desgravación fiscal vinculada a la deuda, debido a la consideración de los intereses como un gasto fiscalmente deducible (Modigliani y Miller, 1963; DeAngelo y Masulis, 1980) y una reducción de los problemas de agencia que surgen entre los accionistas y los directivos con relación al flujo de caja libre (Jensen,1986). Y entre los inconvenientes ligados a la deuda destacan los costes de insolvencia (Myers, 1977) y los costes de agencia que surgen entre los propietarios y prestamistas (Jensen y Meckling, 1976; y Myers, 1977) conforme aumenta el nivel de endeudamiento.

Otra de las corrientes, más reciente que la anterior, para explicar la estructura financiera es la llamada Teoría del Orden de Preferencia o de la Jerarquía entre las fuentes financieras (Pecking order theory), cuyo desarrollo originario se debió a las aportaciones de Myers (1984) y Myers y Majulf (1984). Ellos asumían que los mercados financieros eran perfectos, a excepción de la existencia de información asimétrica, de manera que el hecho de que los directivos, sepan más sobre las expectativas y valores de su empresa que los inversores externos, en ocasiones puede condicionar las decisiones de financiación empresarial.

Para explicar esta teoría se parte de una empresa que necesita recursos externos para financiar una inversión y, en la que existen, entre los gerentes e inversores externos, problemas de información asimétrica con relación a las expectativas de rentabilidad de la nueva inversión y, por tanto, con relación a la valoración de los títulos de la empresa. En este contexto y admitiendo que la alta dirección persigue la maximización de la riqueza de los actuales accionistas, la gerencia deberá elegir entre una emisión de acciones o una de deuda para cubrir la necesidad financiera surgida. En el caso de que la nueva oportunidad de inversión sea buena, a la dirección no le va a interesar emitir acciones si los problemas de información obligan a fijar un precio de emisión por debajo de su valor, porque ello perjudicaría a los actuales accionistas. Si por el contrario las esperanzas con relación a la nueva inversión no fueran tan buenas, el gerente también se vería obligado a renunciar a emitir capital porque un intento de emitir acciones reflejaría a los inversores externos, a consecuencia de los problemas de información asimétrica, que las acciones deberían valer aún menos. Esto origina que las emisiones de acciones en los mercados sean más caras que otro tipo de financiación, porque 
Revista Eletrônica de Ciência Administrativa (RECADM) - ISSN 1677-7387

Faculdade Cenecista de Campo Largo - Coordenação do Curso de Administração

v. 4, n. 1, maio/2005 - http://revistas.facecla.com.br/index.php/recadm/

aparte de los costes de emisión y transacción, las empresas tienen que aceptar el incremento de los costes derivados de la existencia de información asimétrica ${ }^{2}$, y además, como los inversores son conscientes de los problemas derivados de la información asimétrica, el precio de las acciones suele descender ante un anuncio de ampliación de capital a causa de las señales negativas que tales emisiones transfieren a los mismos.

Por lo tanto si existen problemas de información asimétrica y los inversores son racionales, la empresa preferirá, siempre que pueda, las emisiones de deuda a las de acciones. Esta idea se ve también reforzada porque los problemas de información asimétrica con relación a la deuda son mucho menores. Por ello el anuncio de una emisión de deuda tiene un menor impacto negativo sobre la cotización de las acciones a no ser que la deuda sea muy costosa, por ejemplo, porque eleve demasiado el riesgo de insolvencia de la empresa. Obviamente si se utiliza financiación interna los problemas derivados de la información asimétrica son irrelevantes.

En consecuencia la teoría del orden de preferencia no reconoce la existencia de un ratio de endeudamiento óptimo, sino que debido a los problemas de información asimétrica asociados a la financiación externa, las empresas ajustan sus decisiones de financiación a una jerarquía. En primer lugar las empresas prefieren financiación interna a externa. En segundo lugar, y solo si los fondos internos son insuficientes se recurrirá a los fondos externos. Entre los recursos externos prefieren la financiación ajena (primero sin coste, luego a largo plazo, y finalmente emisiones de obligaciones convertibles), dejando la emisión de fondos propios como última alternativa para la obtención de recursos.

Esta breve revisión de las teorías más importantes sobre la estructura financiera empresarial permite identificar múltiples aspectos sobre los que sería interesante detenerse a nivel empírico. Como nuestro objetivo es el estudio de la estructura financiera de las empresas de la Comunidad Autónoma Andaluza,

\footnotetext{
${ }^{2}$ Ya sean costes directos en los que incurriría la empresa para proporcionar una información muy exhaustiva sobre los planes y expectativas futuras de la empresa que permita eliminar los problemas de información asimétrica, objetivo que en la práctica es muy complicado porque resulta muy caro y además es muy difícil superar esas reticencias de los inversores externos; o costes indirectos derivados de fijar unos precios de emisión de las acciones menores a los deseados por la empresa lo que encarece bastante el coste de los capitales propios de la empresa.
} 


\section{Revista Eletrônica de Ciência Administrativa (RECADM) - ISSN 1677-7387 \\ Faculdade Cenecista de Campo Largo - Coordenação do Curso de Administração v. 4, n. 1, maio/2005 - http://revistas.facecla.com.br/index.php/recadm/}

creemos interesante en una primera aproximación plantearnos la influencia de dos factores a los que tradicionalmente se les ha reconocido una cierta importancia a la hora de explicar las decisiones de financiación: el sector de pertenencia de la empresa y el tamaño de la empresa. Ya Harris y Raviv (1991), en la revisión que hacían de los estudios teóricos y empíricos sobre la estructura financiera señalaban que el sector y el tamaño eran algunas de las características específicas de las empresas que se habían considerado como posibles determinantes del nivel de endeudamiento empresarial.

Por un lado y con relación al sector podemos encontrar argumentos que justifiquen su conexión con el endeudamiento de la empresa especialmente dentro de la teoría del óptimo financiero. En este sentido la importancia del sector radica en que las empresas que pertenecen a un mismo campo de actividad deberían presentar una estructura financiera más similar, debido a que de sector a sector varía el riesgo económico, la composición de los activos y las necesidades de recursos. Todavía hoy, y siguiendo a Brealey y Myers (2003), en la realidad se observan diferencias en la estructura de capital entre sectores, de manera que sectores donde los activos sean mayoritariamente intangibles y con mucho riesgo se suelen endeudar poco, frente a sectores donde los activos son tangibles y relativamente seguros. Por este motivo, queremos estudiar si efectivamente la pertenencia a un sector marca diferencias significativas en la estructura financiera de las empresas andaluzas, o si por el contrario y tal como postula la teoría del orden de preferencia (Myers, 1984) el sector no es demasiado relevante sino que son las necesidades de fondos acumuladas por cada empresa a lo largo del tiempo lo que define la composición de su pasivo.

Por otro lado y con relación al factor tamaño son varios los motivos que se han ido argumentando para justificar su conexión con el endeudamiento, algunos apoyados en la corriente del óptimo financiero y otros en la de las preferencias ${ }^{3}$. De

\footnotetext{
${ }^{3}$ La consideración del tamaño con relación a la estructura financiera de las empresas ha ido un poco más allá de lo que representa una simple enumeración de los factores que la condicionan, para llegar al desarrollo de teorías financieras específicas según el tamaño de la empresa. Si bien es cierto que las teorías sobre la estructura financiera previamente mencionadas son en principio válidas para cualquier tipo de empresa ya sea grande o PYME, también es cierto que normalmente los distintos modelos teóricos se han explicado y testado tomando como empresa tipo una grande y cotizada. Esto ha originado en los últimos años una necesidad de adaptación de las teorías financieras a las PYMES, como reconocimiento por parte del colectivo científico del papel preponderante que las pequeñas y medianas empresas han jugado y juegan en el desarrollo económico de muchos países, entre ellos España, y de los problemas de estructura financiera que presentan las empresas de reducida dimensión, lo que lastra, en muchos casos, su desarrollo. Este ha sido el caso de la teoría del orden de preferencia que utilizando
} 


\section{Revista Eletrônica de Ciência Administrativa (RECADM) - ISSN 1677-7387 \\ Faculdade Cenecista de Campo Largo - Coordenação do Curso de Administração v. 4, n. 1, maio/2005 - http://revistas.facecla.com.br/index.php/recadm/}

manera resumida podemos decir que el tamaño afecta a los costes de insolvencia, a los costes de emisión, a los costes de agencia y a los problemas de información asimétrica:

- Costes de insolvencia: en este sentido ya Warner (1977) y Ang, Chua y McConnell (1982) señalaban que a mayor tamaño menor porcentaje representan los costes directos de la quiebra sobre el valor de empresa. Además las empresas relativamente pequeñas debido entre otros a problemas de escasa diversificación y carencias de los gestores tienen una mayor probabilidad de fracaso (Ang, 1991). De hecho y según Rajan y Zingales (1995), el tamaño se puede tomar como una proxy inversa de la probabilidad de insolvencia de una empresa Estos argumentos sugieren que las empresas grandes deberían estar más apalancadas que las pequeñas.

- Costes de emisión de las fuentes: conforme aumenta la dimensión de la empresa aumenta posiblemente también el volumen de las emisiones, lo que permitirá gracias a las economías de escala reducir la significación de los costes de transacción fijos vinculados a las mismas (Scherer y Ross, 1990). Por este motivo a las PYMES no les interesa emitir acciones, porque les sale muy caro, ni tampoco deuda a largo plazo, cuyos costes de formalización son también elevados. Esto parece implicar que las empresas pequeñas posiblemente estén más endeudadas que las grandes, y además que prefieran endeudarse más a corto plazo que a largo plazo debido a los mayores costes fijos asociados a esta última alternativa.

- Costes de agencia. Los problemas de agencia entre las PYMES y las grandes empresas son distintos (Berger y Udell, 1998) ya que mientras en las grandes uno de los problemas de agencia más importante es el que se da entre los directivos y los accionistas, en las PYMES la yuxtaposición de la figura de propietario y gestor hace que este problema esté resuelto. Además, en las PYMES los problemas de agencia que surgen entre ese propietario gestor y los otros stakeholders son mucho más complejos (Ang, 1991) que en las grandes corporaciones. Siguiendo a Ang (1991), esto podría acarrear para las

argumentos específicos para las PYMES, ha dado lugar a una versión modificada tal como la planteó Holmes y Kent (1991), o Ang (1992), debido a las mayores dificultades de las PYMES para acceder a financiación externa. 
Revista Eletrônica de Ciência Administrativa (RECADM) - ISSN 1677-7387 Faculdade Cenecista de Campo Largo - Coordenação do Curso de Administração v. 4, n. 1, maio/2005 - http://revistas.facecla.com.br/index.php/recadm/

PYMES la asunción de mayores riesgos en la gestión, condicionar sus emisiones a motivaciones de mantenimiento del control, imponer una visión cortoplacista en la gestión de la empresa etc. En definitiva estas diferencias en los problemas y, por tanto, en los costes de agencia entre las PYMES y las grandes se traduciría también en diferencias en las decisiones de financiación.

- Información asimétrica: por último el tamaño también puede ser un indicador de los problemas de información asimétrica entre los gerentes y los inversores externos (Rajan y Zingales, 1995). De manera que cuanto más grande sea una empresa menores serán sus problemas de información debido a la mayor calidad y fiabilidad de la información que transmiten a los inversores externos lo que facilitaría las emisiones de acciones, y reduciría las de deuda. Además en las PYMES al ser más fuertes sus problemas de información asimétrica surgen las tradicionales restricciones financieras de los intermediaros financieros para aportar recursos a estas empresas (Hellmann y Stiglitz, 2000).

El efecto de todos estos factores nos lleva a concluir que la relación del tamaño con el nivel de deuda es ambigua tal como ya comentaban Rajan y Zingales (1995). Por un lado cuanto más grande sea una compañía menor será su probabilidad de insolvencia, menores sus costes de agencia de manera que el tamaño tendría un impacto positivo en su nivel de deuda. Sin embargo el tamaño también podría mejorar los problemas de información asimétrica que la empresa tiene con los inversores externos, y reducir los costes de emisión lo que podría incrementar las preferencias de las grandes empresas por las emisiones de acciones frente a las de deuda.

En España se han realizado en los últimos tiempos diversos estudios empíricos sobre la estructura financiera de la empresa española, y en algunos de ellos se ha tenido en cuenta de manera más o menos directa las relaciones entre el tamaño y el endeudamiento, y entre este último y el sector. Entre ellos podemos citar a Ocaña, Salas y Vallés, (1994) que no encuentran una relación sistemática entre el tamaño y el endeudamiento en el sentido que las empresas por clases de tamaño presentan 
Revista Eletrônica de Ciência Administrativa (RECADM) - ISSN 1677-7387

Faculdade Cenecista de Campo Largo - Coordenação do Curso de Administração

v. 4, n. 1, maio/2005 - http://revistas.facecla.com.br/index.php/recadm/

diferencias en la composición de su deuda, pero son mucho más homogéneas en el nivel de endeudamiento. En otros estudios como el de Mato (1990), Fariñas y otros (1992), y Menéndez Requejo (2001) se confirma que las PYMES están significativamente más endeudadas que las grandes. Además en el estudio de Menéndez Requejo (2001) el ratio de endeudamiento sectorial es un determinante de la estructura financiera de las empresas. Por último, Melle (2001) observa que el tamaño y el sector son relevantes para explicar las diferencias en la estructura financiera de las empresas.

Existen, por otro lado, estudios referidos a empresas pertenecientes a una sola clase de tamaño. Entre los estudios que se centran exclusivamente en las PYMES tenemos: Aybar-Arias y López-Gracia (2000) que testan que el tamaño influencia la estrategia de financiación y el sector influencia la política de financiación a corto plazo; Cardone y Casasola (2004) y López-Gracia y Sogrob-Mira (2003), que encuentran una correlación positiva entre la deuda y el tamaño, resultado que no parece confirmarse en Aybar-Arias, Casino, y López-Gracia (2003), sino más bien todo lo contrario. Además, y sobre este mismo grupo de empresas, la relación específica entre el tamaño y la composición de la deuda ha generado resultados contradictorios, y mientras Cardone y Casasola (2004) contrastan que la financiación ajena a corto plazo aumenta con el tamaño, García y Martínez (2004) evidencian lo contrario, es decir que el nivel de endeudamiento a corto plazo es mayor en las empresas pequeñas. Por último, y entre trabajos que consideren sólo a grandes empresas podemos citar a Saá (1991), donde testa que el tamaño no tiene efecto sobre el endeudamiento, o Cuñat (1999) que contrasta que entre el nivel y el plazo de endeudamiento y el tamaño existe una correlación positiva.

Esta diversidad de resultados de los trabajos empíricos viene a confirmar esa ya apuntada ambigüedad de la relación entre de estos dos factores: tamaño (Rajan y Zingales, 1995) y sector (Hall, Hutchinson y Michaelas, 2000) sobre el endeudamiento, debido a las complejas relaciones que los vinculan. Todo ello nos anima a contrastar si el tamaño y el sector son factores explicativos del nivel de endeudamiento de las empresas andaluzas.

\section{ANÁLISIS EMPÍRCO}


Revista Eletrônica de Ciência Administrativa (RECADM) - ISSN 1677-7387

Faculdade Cenecista de Campo Largo - Coordenação do Curso de Administração

v. 4, n. 1, maio/2005 - http://revistas.facecla.com.br/index.php/recadm/

\subsection{MUESTRA UTILIZADA Y VARIABLES RELEVANTES}

Las empresas utilizadas para realizar el estudio empírico las hemos tomado de la base de datos SABI (Sistema de Análisis de Balances Ibéricos). Dicha base de datos recoge información general y las cuentas anuales de más de 550.000 empresas españolas y 50.000 empresas portuguesas. Dentro de España, cubre más del 95\% de las compañías de las 17 Comunidades Autónomas que presentan sus cuentas en registros mercantiles con facturación superior a los 360.000 euros.

Las empresas seleccionadas pertenecen exclusivamente a la Comunidad Autónoma Andaluza, y en la SABI se registran los datos de 80.700 empresas andaluzas. Para la obtención de la muestra definitiva, se tuvieron en cuenta todas las empresas de las que se disponía información para el año 2001, pero los datos fueron filtrados al objeto de eliminar posibles anomalías que distorsionaran los resultados. De manera que no fueron incluidas a) las empresas que no aportaban información suficiente en sus balances; b) las microempresas, esto es empresas que contaban con un número de empleados inferior a dos; c) compañías que presentaran recursos propios negativos.

Para la realización del estudio era necesario que las empresas fueran agrupadas primeramente en función del tamaño al que pertenecen. Para clasificar las empresas en grandes, medianas y pequeñas hemos tenido en cuenta los criterios que se derivan de la Recomendación de la Comisión Europea de 3 de abril de 1996 (Diario Oficial CE [DOCE L 107 de 30/04/1996]), que tendrá vigencia hasta el 31 de diciembre de 2004 y de la Recomendación de la Comisión Europea de 6 de mayo de 2003 que tiene como objetivo redefinir los conceptos de microempresa y PYMEs en el nuevo entorno económico, conceptos que se comenzarán a utilizar a partir del 1 de enero de 2005. En este sentido las características que definen cada grupo son las siguientes:

- Empresas grandes, son las que tienen un número de empleados mayor o igual a 250, las ventas superan o igualan los 40 millones de euros y el volumen de activo es superior o igual a 27 millones de euros.

- Empresas medianas, son las que tienen un número de empleados comprendido entre 249 y 20, las ventas, a su vez, deben estar entre 40 y 7 
Revista Eletrônica de Ciência Administrativa (RECADM) - ISSN 1677-7387

Faculdade Cenecista de Campo Largo - Coordenação do Curso de Administração

v. 4, n. 1, maio/2005 - http://revistas.facecla.com.br/index.php/recadm/

millones de euros, y el volumen de activo debe estar entre 27 y 5 millones de euros.

- Empresas pequeñas, son las que tienen menos de 20 empleados, las ventas deben ser menores a los 7 millones de euros y el volumen de activo no superar los cinco millones de euros.

En nuestro trabajo para incluir a una empresa en uno de los grupos hemos exigido que cumpla dos de los tres criterios definidos anteriormente, y no sólo en el año 2001, sino en los dos precedentes, al objeto de darle más estabilidad a la variable dimensión. Es decir, aquéllas compañías que no permanecían en un mismo grupo de tamaño durante, al menos, tres años consecutivos -1999, 2000, 2001- han sido excluidas de la muestra.

Después de todo este proceso nuestra muestra definitiva está formada por un total de 6.338 empresas andaluzas, de las que 5.606 son pequeñas, 691 medianas y 41 grandes, lo que implica, dado el elevado número de empresas consideradas, una gran cobertura de la muestra. Además, el hecho de que las corporaciones pequeñas representen un $88 \%$ del total de las empresas consideradas, las medianas un 11\%, y las grandes tan sólo un $0,64 \%$, es un fiel reflejo, como no podía ser de otra manera, del tejido empresarial andaluz, en el que predominan las empresas de reducido tamaño ${ }^{4}$.

Por último, nuestra muestra también debía ser clasificada en función del sector de pertenencia. Este factor diferenciador plantea muchos menos problemas en la practica que el factor tamaño. Con relación al sector de actividad hemos utilizado la clasificación sectorial BACH y su correspondencia con la CNAE-93 con dos dígitos ${ }^{5}$ (Tabla 1).

Tabla 1. Clasificación sectorial BACH y su correspondencia con la CNAE

\begin{tabular}{|l|l|}
\hline SECTORES BACH & CNAE-93 \\
\hline Productos energéticos & $10-12,23,40,41$ \\
\hline Industria manufacturera & $13-22,24-26,27-37$ \\
\hline Construcción & 45 \\
\hline
\end{tabular}

\footnotetext{
${ }^{4}$ Entre otras publicaciones que confirman tal dato podemos citar a FUNCAS (2003): Las PYME españolas con forma societaria. Estructura económico financiero y resultados. Madrid: Ministerio de Economía.

${ }^{5}$ Recordamos que la base de datos SABI no incluye las empresas financieras y de seguros.
} 
Revista Eletrônica de Ciência Administrativa (RECADM) - ISSN 1677-7387 Faculdade Cenecista de Campo Largo - Coordenação do Curso de Administração v. 4, n. 1, maio/2005 - http://revistas.facecla.com.br/index.php/recadm/

\begin{tabular}{|l|l|}
\hline Servicios & $\begin{array}{l}50-52,55,60-64,70-74,80,85, \\
90,92,93\end{array}$ \\
\hline Agricultura y Ganadería & $01,02,05$ \\
\hline
\end{tabular}

La distribución sectorial de las empresas que configuran la muestra por sector de actividad aparece en la Tabla 2.

Tabla 2. Porcentaje de empresas de la muestra por sector de actividad

\begin{tabular}{|l|l|c|}
\hline SECTORES & ACTIVIDAD & $\begin{array}{l}\text { IMPORTANCIA RELATIVA } \\
\mathbf{( \% )}\end{array}$ \\
\hline Sector 1 & $\begin{array}{l}\text { Agricultura } \\
\text { ganadería }\end{array}$ & 2,61 \\
\hline Sector 2 & Construcción & 10,5 \\
\hline Sector 3 & Energía & 0,84 \\
\hline Sector 4 & Manufactura & 17,83 \\
\hline Sector 5 & Servicios & 68,22 \\
\hline
\end{tabular}

Finalmente, la población utilizada en función de los criterios anteriormente definidos la podemos resumir en la tabla 3.

Tabla 3. Muestra de empresas andaluzas clasificadas por sectores y tamaños.

\begin{tabular}{|l|r|r|r|r|}
\hline SECTOR Y TAMAÑO & GRANDE & MEDIANA & PEQUEÑA & \multicolumn{1}{l|}{ TOTAL } \\
\hline Sector 1 & 3 & 30 & 134 & 167 \\
\hline Sector 2 & 4 & 71 & 591 & 666 \\
\hline Sector 3 & 1 & 13 & 39 & 53 \\
\hline Sector 4 & 15 & 167 & 948 & 1.130 \\
\hline Sector 5 & 18 & 410 & 3.894 & 4.322 \\
\hline
\end{tabular}


Revista Eletrônica de Ciência Administrativa (RECADM) - ISSN 1677-7387

Faculdade Cenecista de Campo Largo - Coordenação do Curso de Administração

v. 4, n. 1, maio/2005 - http://revistas.facecla.com.br/index.php/recadm/

\begin{tabular}{|l|r|r|r|r|}
\hline TOTAL & 41 & 691 & 5.606 & 6.338 \\
\hline
\end{tabular}

Por último para identificar la importancia de estos dos factores: tamaño y sector, sobre la estructura financiera debemos definir una serie de ratios que definan la misma. En la Teoría Financiera existen distintos indicadores que definen el nivel de financiación de una empresa. Después de analizar los últimos trabajos orientados a la estructura de capital y, fundamentalmente, por el tipo de información disponible hemos decidido optar por la relación existente entre los recursos ajenos y los recursos totales como indicador representativo del nivel de endeudamiento de la empresa. Además y para aprovechar la información disponible hemos incluido dos ratios más: recursos ajenos a corto plazo entre recursos totales y recursos ajenos a largo plazo entre recursos totales. Gracias a estas dos últimas variables, aparte de analizar de manera genérica la estructura financiera de las empresas andaluzas, recogeremos aspectos relativos a la composición y plazos de los recursos ajenos.

\subsection{ANÁLISIS DESCRIPTIVO}

Iniciamos nuestro estudio con el análisis de las principales variables descriptoras de la estructura financiera de la empresa andaluza. Este análisis es puramente descriptivo, y se desarrollará a través de la utilización de una serie de estadísticos sobre las variables definidas anteriormente. El examen se realizará estructurando dichas variables en función del tamaño y el sector de actividad al que pertenezcan las empresas.

En primer lugar, destaca que la estructura financiera de la empresa andaluza presenta, en general, un elevado nivel de endeudamiento, en torno al $70 \%$ de sus recursos son ajenos (tabla 4). Si este estudio lo realizamos teniendo en cuenta el tamaño, son las empresas pequeñas las que tienen un mayor nivel de deuda, un $72 \%$ frente a un $65 \%$ y un $68 \%$ que ofrecen las empresas medianas y grandes, respectivamente. Estos resultados coinciden con el estudio de Funcas $(2003)^{6}$ y Maroto(1996) ${ }^{7}$.

\footnotetext{
${ }^{6}$ En este estudio y para España en el año 2001 son las empresas medianas las que presentan menores niveles de endeudamiento (sobre un 59\%), mientras que las grandes y las pequeñas tienen mayores niveles de deuda (se aproximan al 70\%).

${ }^{7}$ Aunque el análisis de Maroto (1996) está referido a la situación financiera de las empresas españolas en la primera mitad de los años noventa y toma la información de la Central de Balances del Banco de España.
} 
Revista Eletrônica de Ciência Administrativa (RECADM) - ISSN 1677-7387

Faculdade Cenecista de Campo Largo - Coordenação do Curso de Administração v. 4, n. 1, maio/2005 - http://revistas.facecla.com.br/index.php/recadm/

Por otro lado, hay que señalar que el origen de tales diferencias se encuentra en los recursos ajenos a corto plazo más que en el endeudamiento a largo plazo, donde, en términos medios, parece que el comportamiento de las empresas es más uniforme.

La fiabilidad de los valores obtenidos para las empresas medianas y pequeñas podemos considerarla aceptable ya que las desviaciones típicas no son excesivamente altas, al estar, en la mayor parte de los casos, la media y mediana muy próximas entre sí. Además, si observamos el gráfico de frecuencias presentado en la figura 1 comprobamos como la mayor densidad de empresas se encuentra en la parte derecha, que es la zona de mayor endeudamiento. Respecto a los resultados obtenidos en las empresas grandes hay que tomarlos con cautela, ya que el número de éstas en la Comunidad Andaluza es muy pequeño y, por lo tanto, pueden no ser representativos.

Tabla 4. Estadísticos descriptivos de las empresas andaluzas clasificadas por tamaño.

\begin{tabular}{|c|c|c|c|c|c|c|c|c|c|}
\hline \multirow{2}{*}{$\begin{array}{c}\text { TAMAÑ } \\
0\end{array}$} & \multicolumn{3}{|c|}{$\begin{array}{c}\text { REC AJENOS/REC } \\
\text { TOT }\end{array}$} & \multicolumn{3}{|c|}{$\begin{array}{c}\text { REC AJENOS I/p I } \\
\text { R T }\end{array}$} & \multicolumn{3}{|c|}{$\begin{array}{c}\text { REC AJENOS c/p l } \\
\text { R T }\end{array}$} \\
\hline & $\begin{array}{c}\text { Med } \\
\text { ia }\end{array}$ & $\begin{array}{c}\text { Desviac } \\
\text { ión } \\
\text { típica }\end{array}$ & $\begin{array}{c}\text { Medi } \\
\text { an }\end{array}$ & $\begin{array}{c}\text { Med } \\
\text { ia }\end{array}$ & $\begin{array}{c}\text { Desviac } \\
\text { ión } \\
\text { típica }\end{array}$ & $\begin{array}{c}\text { Medi } \\
\text { an }\end{array}$ & $\begin{array}{c}\text { Med } \\
\text { ia }\end{array}$ & $\begin{array}{c}\text { Desviac } \\
\text { ión } \\
\text { típica }\end{array}$ & \begin{tabular}{|c|} 
Medi \\
an
\end{tabular} \\
\hline $\begin{array}{l}\text { 1(peque } \\
\text { ña) }\end{array}$ & $\begin{array}{r}, 720 \\
5\end{array}$ & 316 & 7800 & $\begin{array}{r}, 138 \\
5\end{array}$ & 16815, & ,0700 & $\begin{array}{r}, 582 \\
0\end{array}$ & 23813, & |6100 \\
\hline $\begin{array}{l}\text { 2(media } \\
\text { na) }\end{array}$ & $\begin{array}{r}, 652 \\
4\end{array}$ & 22974, & 7000 & $\begin{array}{r}, 131 \\
1\end{array}$ & 16321, & ,0700 & $\begin{array}{r}, 521 \\
6\end{array}$ & 24314, & ,5300 \\
\hline $\begin{array}{c}\text { 3(grand } \\
\text { e) }\end{array}$ & $\begin{array}{r}, 684 \\
1\end{array}$ & 21526, & 7300 & $\begin{array}{r}, 145 \\
6\end{array}$ & 18941, & , 0800 & $\begin{array}{r}, 538 \\
8\end{array}$ & 25838, & \\
\hline TOTAL & $\begin{array}{r}, 717 \\
3\end{array}$ & 22573, & & $\begin{array}{r}, 138 \\
8\end{array}$ & 1691, & & $\begin{array}{r}, 578 \\
4\end{array}$ & 24088, & \\
\hline
\end{tabular}

Figura 1. Histograma de la estructura financiera por grupos de tamaño.

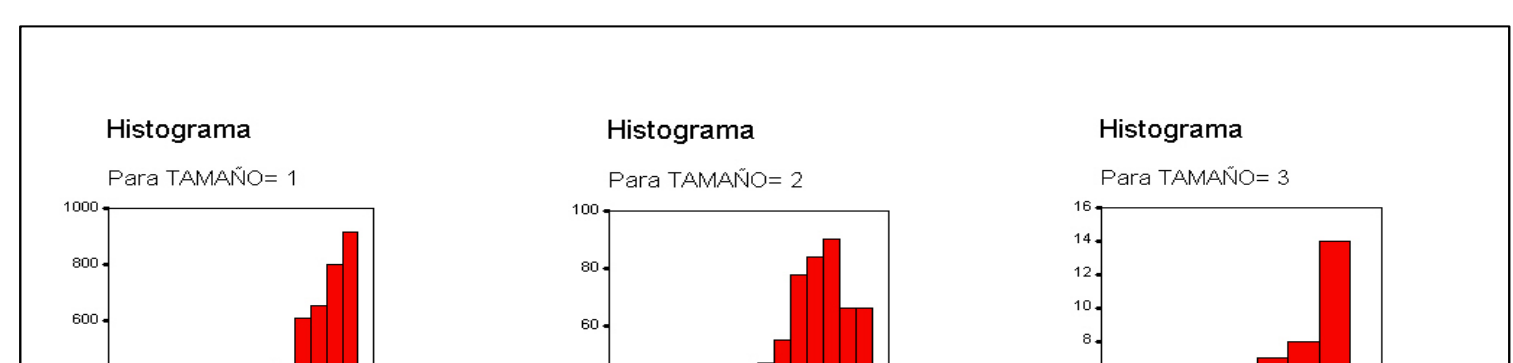


Revista Eletrônica de Ciência Administrativa (RECADM) - ISSN 1677-7387

Faculdade Cenecista de Campo Largo - Coordenação do Curso de Administração

v. 4, n. 1, maio/2005 - http://revistas.facecla.com.br/index.php/recadm/

Si analizamos los percentiles de los ratios financieros según el tamaño (tabla 5) comprobamos como solo el 10\% de las empresas pequeñas en Andalucía poseen una estructura financiera donde predominan los recursos propios sobre los ajenos. Sin embargo, en las empresas medianas este porcentaje sube a casi un $25 \%$. Además, la distancia entre el percentil 10 y el 50 es dos veces mayor a la que hay entre el percentil 50 y 90, hecho que nos vuelva a confirmar la mayor concentración de empresas en la zona de máximo endeudamiento. Si observamos la composición de los recursos ajenos volvemos a comprobar que el corto plazo prevalece por encima del largo plazo. Así, el $25 \%$ de las empresas andaluzas no tienen deuda a largo plazo y el 50\% poseen una ratio de deuda a largo plazo sobre recursos totales del $7 \%$.

Tabla 5. Percentiles de la estructura financiera de las empresas andaluzas por tamaño.

\begin{tabular}{|l|c|r|r|r|r|r|r|r|}
\hline \multicolumn{9}{|c|}{ PERCENTILES } \\
\hline VARIABLE & TAMAÑO & $\mathbf{5}$ & $\mathbf{1 0}$ & $\mathbf{2 5}$ & $\mathbf{5 0}$ & $\mathbf{7 5}$ & $\mathbf{9 0}$ & $\mathbf{9 5}$ \\
\hline R.AJENOS/RT & $\mathbf{1}$ & 0,26 & 0,39 & 0,60 & 0,78 & 0,90 & 0,95 & 0,97 \\
\hline & $\mathbf{2}$ & 0,18 & 0,29 & 0,51 & 0,70 & 0,83 & 0,92 & 0,94 \\
\hline & $\mathbf{3}$ & 0,27 & 0,35 & 0,55 & 0,73 & 0,87 & 0,90 & 0,94 \\
\hline R A l/p / RT & $\mathbf{1}$ & 0,00 & 0,00 & 0,00 & 0,07 & 0,21 & 0,39 & 0,50 \\
\hline & $\mathbf{2}$ & 0,00 & 0,00 & 0,01 & 0,07 & 0,20 & 0,36 & 0,52 \\
\hline & $\mathbf{3}$ & 0,00 & 0,00 & 0,01 & 0,08 & 0,17 & 0,44 & 0,66 \\
\hline R A c/p / RT & $\mathbf{1}$ & 0,15 & 0,24 & 0,41 & 0,61 & 0,78 & 0,88 & 0,92 \\
\hline & $\mathbf{2}$ & 0,11 & 0,17 & 0,32 & 0,56 & 0,73 & 0,83 & 0,88 \\
\hline & $\mathbf{3}$ & 0,13 & 0,17 & 0,33 & 0,53 & 0,80 & 0,88 & 0,90 \\
\hline
\end{tabular}

En segundo lugar, realizamos el análisis de la estructura financiera de las empresas andaluzas clasificándolas en función del sector de actividad al que pertenecen. En todos los sectores predomina la financiación con recursos ajenos sobre los recursos propios, y dentro de los recursos ajenos sobresale la financiación a corto plazo, al igual que sucedía con el tamaño. En cuanto a los sectores más 
Revista Eletrônica de Ciência Administrativa (RECADM) - ISSN 1677-7387

Faculdade Cenecista de Campo Largo - Coordenação do Curso de Administração v. 4, n. 1, maio/2005 - http://revistas.facecla.com.br/index.php/recadm/

endeudados destacan el de la construcción y el de servicios con unos ratios del 76\% y $72 \%$, respectivamente (tabla 6). De la misma manera, estos sectores son los que poseen un mayor predominio de la financiación a corto plazo ${ }^{8}$. Los sectores menos endeudados y los que presentan un mayor nivel de endeudamiento a largo plazo son el energético y el agrícola, aunque la representatividad de estos sectores en el tamaño de la población es muy pequeña.

Tabla 6. Estadísticos descriptivos de empresas andaluzas clasificadas por sectores de actividad.

\begin{tabular}{|c|c|c|c|c|c|c|c|c|c|}
\hline \multirow{2}{*}{$\begin{array}{l}\text { SECT } \\
\text { OR }\end{array}$} & \multicolumn{3}{|c|}{$\begin{array}{c}\text { REC AJENOS/REC } \\
\text { TOT }\end{array}$} & \multicolumn{3}{|c|}{$\begin{array}{c}\text { REC AJENOS } I / p ~ I ~ \\
\text { R T }\end{array}$} & \multicolumn{3}{|c|}{$\begin{array}{c}\text { REC AJENOS } c / p ~ I \\
\text { R T }\end{array}$} \\
\hline & $\begin{array}{c}\text { Medi } \\
\text { a }\end{array}$ & $\begin{array}{c}\text { Desviac } \\
\text { ión } \\
\text { típica }\end{array}$ & $\begin{array}{l}\text { Medi } \\
\text { an }\end{array}$ & $\begin{array}{c}\text { Medi } \\
\text { a }\end{array}$ & $\begin{array}{c}\text { Desviac } \\
\text { ión } \\
\text { típica }\end{array}$ & $\begin{array}{l}\text { Medi } \\
\text { an }\end{array}$ & $\begin{array}{l}\text { Medi } \\
\text { a }\end{array}$ & $\begin{array}{c}\text { Desviac } \\
\text { ión } \\
\text { típica }\end{array}$ & $\begin{array}{l}\text { Medi } \\
\text { an }\end{array}$ \\
\hline 1 & $\begin{array}{r}, 616 \\
38\end{array}$ & 303742 & ,6400 & $\begin{array}{r}, 150 \\
69\end{array}$ & 187500, & ,0700 & $\begin{array}{r}, 465 \\
69\end{array}$ & ,274591 & ,4400 \\
\hline 2 & $\begin{array}{r}760 \\
90\end{array}$ & 205969, & ,8200 & $\begin{array}{r}122 \\
35\end{array}$ & ,163153 & ,0500 & $\begin{array}{r}638 \\
55\end{array}$ & ,226706 & ,6700 \\
\hline 3 & $\begin{array}{r}, 522 \\
04\end{array}$ & 283154 & ,5450 & $\begin{array}{r}, 154 \\
54\end{array}$ & ,185596 & ,0800 & $\begin{array}{r}, 367 \\
49\end{array}$ & ,238932 & ,3300 \\
\hline 4 & $\begin{array}{r}, 693 \\
51\end{array}$ & 223580 & ,7400 & $\begin{array}{r}, 144 \\
95\end{array}$ & ,154050 & 1000 & $\begin{array}{r}, 548 \\
56\end{array}$ & ,227753 & ,5700 \\
\hline 5 & $\begin{array}{r}, 723 \\
12\end{array}$ & ,222029 & ,7800 & $\begin{array}{r}139 \\
18\end{array}$ & ,172713 & ,0700 & $\begin{array}{r}, 583 \\
94\end{array}$ & ,241226 & 6100 \\
\hline
\end{tabular}

Según los percentiles por sectores de actividad (tabla 7) y sus gráficos de frecuencia (figura 2), comprobamos como en el $20 \%$ de las empresas predominan los recursos propios sobre los ajenos. En el caso del sector energético y agrícola el

\footnotetext{
${ }^{8}$ Los resultados del estudio descriptivo realizado por Cardone y Casasola (2004), para una muestra de PYMES españolas seleccionada a partir de los datos de la base de datos SABI, coinciden con nosotros a la hora de establecer los sectores más endeudados: primero construcción y después servicios, sin embargo los niveles de endeudamiento de las empresas andaluzas por sectores son superiores a las medias estatales.
} 
Revista Eletrônica de Ciência Administrativa (RECADM) - ISSN 1677-7387

Faculdade Cenecista de Campo Largo - Coordenação do Curso de Administração

v. 4, n. 1, maio/2005 - http://revistas.facecla.com.br/index.php/recadm/

$45 \%$ de las empresas poseen un nivel de financiación propia superior a la ajena. En los sectores de construcción y servicios este porcentaje desciende al 15\%. Por otra parte, si examinamos la distancia entre el percentil 10 y 50 y el 50 y 90, observamos como la primera diferencia es mucho más amplia que la segunda, lo que nos vuelve a indicar que el nivel de endeudamiento se estabiliza progresivamente a partir del percentil 50, como ocurría con el tamaño.

Tabla 7. Percentiles de la estructura financiera de las empresas andaluzas clasificadas por sectores.

\begin{tabular}{|c|c|c|c|c|c|c|c|c|}
\hline \multicolumn{9}{|c|}{ PERCENTILES } \\
\hline VARIABLE & SECTOR & 5 & 10 & 25 & 50 & 75 & 90 & 95 \\
\hline \multirow[t]{5}{*}{ RA/RT } & 1 & 0,09 & 0,144 & 0,32 & 0,64 & 0,89 & 0,96 & 0,98 \\
\hline & 2 & 0,334 & 0,44 & 0,66 & 0,82 & 0,92 & 0,96 & 0,97 \\
\hline & 3 & 0,086 & 0,113 & 0,2275 & 0,545 & 0,7775 & 0,909 & 0,9435 \\
\hline & 4 & 0,25 & 0,367 & 0,55 & 0,74 & 0,87 & 0,95 & 0,97 \\
\hline & 5 & 0,26 & 0,38 & 0,6 & 0,78 & 0,89 & 0,95 & 0,97 \\
\hline \multirow[t]{5}{*}{ R A l/p / RT } & 1 & 0 & 0 & 0,01 & 0,07 & 0,22 & 0,44 & 0,576 \\
\hline & 2 & 0 & 0 & 0,01 & 0,05 & 0,17 & 0,35 & 0,463 \\
\hline & 3 & 0 & 0 & 0,01 & 0,08 & 0,2025 & 0,448 & 0,594 \\
\hline & 4 & 0 & 0 & 0,02 & 0,1 & 0,22 & 0,37 & 0,47 \\
\hline & 5 & 0 & 0 & 0 & 0,07 & 0,21 & 0,39 & 0,5 \\
\hline \multirow[t]{5}{*}{ R A c/p / RT } & 1 & 0,06 & 0,1 & 0,22 & 0,44 & 0,66 & 0,852 & 0,898 \\
\hline & 2 & 0,2 & 0,31 & 0,475 & 0,67 & 0,82 & 0,91 & 0,933 \\
\hline & 3 & 0,0565 & 0,09 & 0,18 & 0,33 & 0,5275 & 0,747 & 0,878 \\
\hline & 4 & 0,15 & 0,24 & 0,37 & 0,57 & 0,73 & 0,85 & 0,91 \\
\hline & 5 & 0,14 & 0,23 & 0,4 & 0,61 & 0,78 & 0,87 & 0,92 \\
\hline
\end{tabular}

Finalmente, si combinamos los grupos de empresas por tamaño y sectores y por sectores y tamaños, respectivamente alcanzamos los mismos resultados obtenidos anteriormente, alto nivel de endeudamiento y predominio de la deuda a corto (tabla 8). Sin embargo, si analizamos la combinación entre sectores y tamaño conseguimos nuevas observaciones. En el sector de la construcción cuanto mayor es el tamaño mayor es el endeudamiento y en el sector servicios las empresas grandes son las que mantienen un mayor nivel de de deuda y las medianas las que menos lo están.

Figura 2. Histograma de la estructura financiera por grupos de sectores 
Revista Eletrônica de Ciência Administrativa (RECADM) - ISSN 1677-7387 Faculdade Cenecista de Campo Largo - Coordenação do Curso de Administração v. 4, n. 1, maio/2005 - http://revistas.facecla.com.br/index.php/recadm/

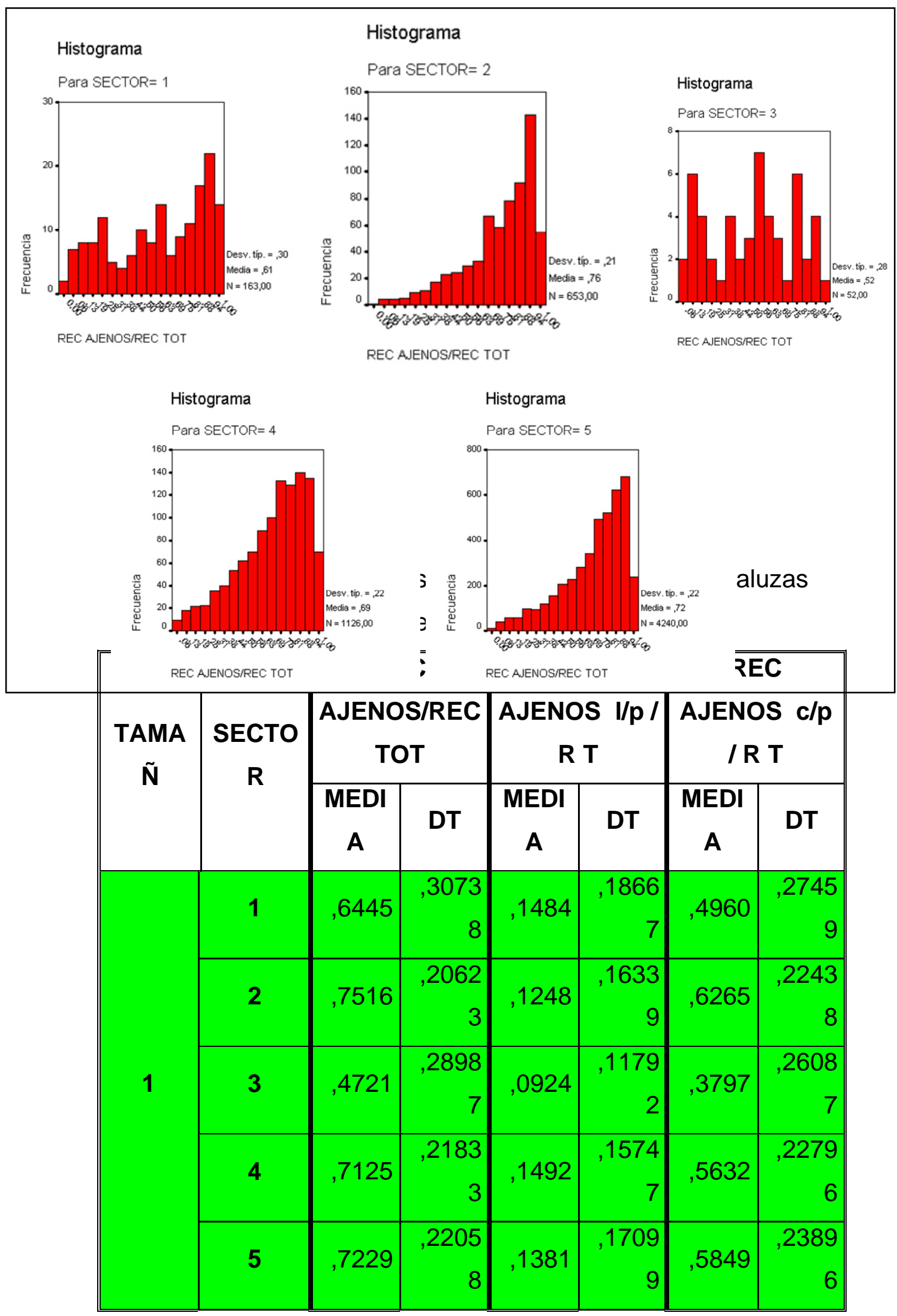


Revista Eletrônica de Ciência Administrativa (RECADM) - ISSN 1677-7387 Faculdade Cenecista de Campo Largo - Coordenação do Curso de Administração v. 4, n. 1, maio/2005 - http://revistas.facecla.com.br/index.php/recadm/

\begin{tabular}{|c|c|c|c|c|c|c|c|}
\hline \multirow{5}{*}{2} & 1 & ,4760 & $\begin{array}{r}2272 \\
0\end{array}$ & ,1677 & $\begin{array}{r}2002 \\
7\end{array}$ & 3087 & $\begin{array}{r}, 1758 \\
7\end{array}$ \\
\hline & 2 & ,7872 & $\begin{array}{r}, 1998 \\
7\end{array}$ & ,1007 & $\begin{array}{r}1544 \\
0\end{array}$ & ,6865 & $\begin{array}{r}, 2268 \\
5\end{array}$ \\
\hline & 3 & ,6446 & $\begin{array}{r}, 2278 \\
7\end{array}$ & ,3123 & $\begin{array}{r}2451 \\
6\end{array}$ & ,3331 & $\begin{array}{r}\text {,1892 } \\
3\end{array}$ \\
\hline & 4 & ,5838 & $\begin{array}{r}2218 \\
1\end{array}$ & 1151 & $\begin{array}{r}1202 \\
8\end{array}$ & ,4691 & $\begin{array}{r}, 2083 \\
9\end{array}$ \\
\hline & 5 & 6703 & $\begin{array}{r}2221 \\
3\end{array}$ & ,1344 & $\begin{array}{r}1702 \\
5\end{array}$ & ,5362 & $\begin{array}{r}, 2440 \\
3\end{array}$ \\
\hline \multirow{5}{*}{3} & 1 & ,3100 & $\begin{array}{r}, 2821 \\
3\end{array}$ & ,0967 & $\begin{array}{r}, 0850 \\
5\end{array}$ & ,2167 & $\begin{array}{r}, 2040 \\
4\end{array}$ \\
\hline & 2 & ,8925 & $\begin{array}{r}, 0275 \\
4\end{array}$ & ,0325 & $\begin{array}{r}, 0330 \\
4\end{array}$ & ,8525 & $\begin{array}{r}, 0525 \\
2\end{array}$ \\
\hline & 3 & ,5000 & , & ,1600 & , & ,3300 & \\
\hline & 4 & ,6433 & $\begin{array}{r}2019 \\
4\end{array}$ & ,1953 & $\begin{array}{r}, 2338 \\
8\end{array}$ & ,4507 & $\begin{array}{r}, 2181 \\
6\end{array}$ \\
\hline & 5 & ,7444 & $\begin{array}{r}, 1539 \\
8\end{array}$ & ,1367 & $\begin{array}{r}1805 \\
9\end{array}$ & ,6078 & $\begin{array}{r}, 2378 \\
7\end{array}$ \\
\hline \multirow{2}{*}{$\begin{array}{c}\text { SECTO } \\
\text { R }\end{array}$} & \multirow[t]{2}{*}{ TAMAÑ } & \multicolumn{2}{|c|}{$\begin{array}{c}\text { REC } \\
\text { AJENOS/RE } \\
\text { C TOT }\end{array}$} & \multicolumn{2}{|c|}{$\begin{array}{c}\text { REC } \\
\text { AJENOS I/p I } \\
\text { R T }\end{array}$} & \multicolumn{2}{|c|}{$\begin{array}{c}\text { REC } \\
\text { AJENOS c/p } \\
\text { I R T }\end{array}$} \\
\hline & & $\begin{array}{c}\text { MEDI } \\
\text { A }\end{array}$ & DT & $\begin{array}{c}\text { MEDI } \\
\text { A }\end{array}$ & DT & $\begin{array}{c}\text { MEDI } \\
\text { A }\end{array}$ & DT \\
\hline \multirow{3}{*}{1} & 1 & ,6445 & $\begin{array}{r}, 3073 \\
8\end{array}$ & ,1484 & $\begin{array}{r}1866 \\
7\end{array}$ & ,4960 & $\begin{array}{r}, 2745 \\
9\end{array}$ \\
\hline & 2 & ,4760 & $\begin{array}{r}, 2272 \\
0\end{array}$ & 1677 & $\begin{array}{r}, 2002 \\
7\end{array}$ & ,3087 & $\begin{array}{r}, 1758 \\
7\end{array}$ \\
\hline & 3 & ,3100 & $\begin{array}{r}, 2821 \\
3\end{array}$ & ,0967 & $\begin{array}{r}, 0850 \\
5\end{array}$ & ,2167 & ,2040 \\
\hline
\end{tabular}


Revista Eletrônica de Ciência Administrativa (RECADM) - ISSN 1677-7387 Faculdade Cenecista de Campo Largo - Coordenação do Curso de Administração v. 4, n. 1, maio/2005 - http://revistas.facecla.com.br/index.php/recadm/

\begin{tabular}{|c|c|c|c|c|c|c|c|}
\hline \multirow{3}{*}{2} & 1 & ,7516 & $\begin{array}{r}, 2062 \\
3\end{array}$ & 1248 & $\begin{array}{r}, 1633 \\
9\end{array}$ & ,6265 & $\begin{array}{r}, 2243 \\
8\end{array}$ \\
\hline & 2 & ,7872 & $\begin{array}{r}1998 \\
7\end{array}$ & 1007, & $\begin{array}{r}1544 \\
0\end{array}$ & 6865 & $\begin{array}{r}, 2268 \\
5\end{array}$ \\
\hline & 3 & 8925, & $\begin{array}{r}, 0275 \\
4\end{array}$ & ,0325 & $\begin{array}{r}, 0330 \\
4\end{array}$ & 8525, & $\begin{array}{r}, 0525 \\
2\end{array}$ \\
\hline \multirow{3}{*}{3} & 1 & ,4721 & $\begin{array}{r}, 2898 \\
7\end{array}$ & ,0924 & $\begin{array}{r}, 1179 \\
2\end{array}$ & ,3797 & $\begin{array}{r}, 2608 \\
7\end{array}$ \\
\hline & 2 & ,6446 & $\begin{array}{r}, 2278 \\
7\end{array}$ & ,3123 & $\begin{array}{r}, 2451 \\
6\end{array}$ & ,3331 & $\begin{array}{r}, 1892 \\
3\end{array}$ \\
\hline & 3 & ,5000 & , & 1600 & , & ,3300 & \\
\hline \multirow{3}{*}{4} & 1 & ,7125 & $\begin{array}{r}, 2183 \\
3\end{array}$ & ,1492 & $\begin{array}{r}, 1574 \\
7\end{array}$ & ,5632 & $\begin{array}{r}, 2279 \\
6\end{array}$ \\
\hline & 2 & ,5838 & $\begin{array}{r}2218 \\
1\end{array}$ & ,1151 & $\begin{array}{r}1202 \\
8\end{array}$ & 4691 & $\begin{array}{r}, 2083 \\
9\end{array}$ \\
\hline & 3 & 6433 & $\begin{array}{r}2019 \\
4\end{array}$ & ,1953 & $\begin{array}{r}, 2338 \\
8\end{array}$ & 4507 & $\begin{array}{r}, 2181 \\
6\end{array}$ \\
\hline \multirow{3}{*}{5} & 1 &, 7229 & $\begin{array}{r}, 2205 \\
8\end{array}$ & ,1381 & $\begin{array}{r}1709 \\
9\end{array}$ &, 5849 & $\begin{array}{r}, 2389 \\
6\end{array}$ \\
\hline & 2 & ,6703 & $\begin{array}{r}, 2221 \\
3\end{array}$ & ,1344 & $\begin{array}{r}1702 \\
5\end{array}$ &, 5362 & $\begin{array}{r}, 2440 \\
3\end{array}$ \\
\hline & 3 & ,7444 & $\begin{array}{r}, 1539 \\
8\end{array}$ & ,1367 & $\begin{array}{r}1805 \\
9\end{array}$ & 6078 & $\begin{array}{r}, 2378 \\
7\end{array}$ \\
\hline
\end{tabular}

\section{CONCLUSIONES}

En esta comunicación hemos realizado un estudio de la estructura financiera de las empresas andaluzas, diferenciando las mismas en función del tamaño y el sector de actividad. Gracias a este trabajo de carácter descriptivo hemos obtenido una serie de consideraciones a cerca de las empresas andaluzas. 
Revista Eletrônica de Ciência Administrativa (RECADM) - ISSN 1677-7387

Faculdade Cenecista de Campo Largo - Coordenação do Curso de Administração v. 4, n. 1, maio/2005 - http://revistas.facecla.com.br/index.php/recadm/

Con carácter general comprobamos que las empresas andaluzas están muy endeudadas, alcanzando un nivel de endeudamiento del 70\%. Además, debemos añadir que es la financiación ajena a corto plazo el principal origen de la deuda.

En cuanto al tamaño, la pequeña empresa destaca por su mayor nivel de apalancamiento, siendo la mediana la menos endeudada, y colocándose la gran empresa en un nivel medio. De nuevo observamos que en todos los tamaños la composición de la deuda es prioritariamente a corto, aunque en la pequeña es más importante.

Por sectores de actividad, el de construcción y servicios son los más endeudados $y$, además, su financiación es fundamentalmente a corto plazo. Los sectores menos endeudados y los que presentan un mayor nivel de endeudamiento a largo plazo son el energético y el agrícola, aunque la representatividad de estos sectores en el tamaño de la población es muy pequeña.

Finalmente y después de analizar los distintos estadísticos descriptivos sobre la estructura financiera andaluza en relación al tamaño y sector de actividad no hemos encontrado una relación lo suficientemente representativa que nos indique una dependencia entre la financiación de la empresa andaluza y el tamaño o el sector donde desempeña su actividad.

\section{BIBLIOGRAFÍA}

ANG,J. (1992): "On the theory of finance for privately held firms", Journal of Small Business Finance, 1, 3, pp. 185-203.

ANG, J. (1991): "Small business uniqueness and the theory of financial management", Journal of Small Business Finance, 1,1, pp. 1-13.

ANG, J.; CHUA,J. y MCCONNELL, J. (1982): "The administrative costs of business bankruptcy", Journal of Finance.

BERGER, A. y UDELL, G. (1998): "The economics of small business finance: the roles of private equity and debt markets in the financial growth cycle", Journal of Banking \& Finance, 22, pp. 613-673.

BREALEY, R. y MYERS, S. (2003): Fundamentos de financiación empresarial, $7^{\text {a }}$ ed. McGraw Hill, Madrid. 
Revista Eletrônica de Ciência Administrativa (RECADM) - ISSN 1677-7387

Faculdade Cenecista de Campo Largo - Coordenação do Curso de Administração

v. 4, n. 1, maio/2005 - http://revistas.facecla.com.br/index.php/recadm/

BOEDO, L. y CALVO, A.R. (1997): "Un modelo de síntesis de los factores que detrerminan la estructura de capital óptima de las PYMES", Revista Europea de Dirección y Economía de la Empresa, $\mathrm{n}^{\circ}$ 1, pp. 107-124.

CARDONE-RIPORTELLA, C. y CASSASOLA MARTÍNEZ, M.J. (2004): "Comportamiento financiero de la PYME española: una visión desde la teoría del orden de preferencias", XII Foro de Finanzas, Barcelona, diciembre.

CUÑAT, V. (1999): "Determinantes del plazo de endeudamiento de las empresas españolas", Investigaciones Económicas, 23, pp. 351-392.

DeANGELO, H. y MASULIS, R. (1980): "Optimal capital structure Ander corporate and personal taxation", Journal of Financial Economics, 8, pp. 451-471.

DE MIGUEL, A. y PINDADO, J. (2001): "Determinants of capital structure: new evidence from Spanish panel data"; Journal of Corporate Finance, 7, pp. 77-99.

FAMA, E. y FRENCH, K. (2002): "Testing trade-off and pecking order predictions about dividends and debt", The Review of Financial Studies, 15, 1, pp. 1-33.

FARIÑAS y OTROS (1992): La PYME industrial en España, Madrid: Civitas.

FUNCÁS (2003); Las PYMES españolas con forma societaria, Ministerio de Economía, Madrid.

GARCÍA TERUEL, P. y MARTíNEZ SOLANO, P. (2004): "Endeudamiento a corto plazo en las PYMES españolas", XII Foro de Finanzas, Diciembre, Barcelona.

HALL, G.; HUTCHINSON, P. y MICHAELAS, N. (2000): "Industry effects on the determinants of unquotted SMEs' capital structure", International Journal of the Economics Business, 7, 3,pp. 297-312.

HARRIS, M. y RAVIV, A. (1991): "The theory of capital structure", Journal of Finance, 46, 1, pp. 297-355.

HELLMANN, T. y STIGLITZ, J. (2000): "Credit and equity rationing in markets with adverse selection", European Economic Review, 44, pp. 281-304.

HOLMES, S. y KENT, P. (1991): "An empirical analysis of the financial structure of small and large australian manufacturing enterprises", Journal of Small Business Finance, 1,2, pp. 141-154.

JENSEN, M. (1986): "Agency costs of free cash-flow, corporate finance and takeovers" American Economic Review, 76, 2, pp. 323-329. 
Revista Eletrônica de Ciência Administrativa (RECADM) - ISSN 1677-7387

Faculdade Cenecista de Campo Largo - Coordenação do Curso de Administração

v. 4, n. 1, maio/2005 - http://revistas.facecla.com.br/index.php/recadm/

JENSEN, M. y MECKLING, W. (1976): "Theory of the firm: managerial behaviour, agency costs and ownership structure", Journal of Financial Economics, 3,4, pp. 305360.

LÓPEZ-GRACIA, J. y AYBAR-ARIAS, C. (2000): "An empirical approach to the financial behaviour of small and medium sized companies", Small Business Economics, 14, pp. 55-63.

LÓPEZ-GRACIA, J.; AYBAR-ARIAS, C. y CASINO MARTÍNEZ (2003): "Estrategia y estructura de capital en la PYME: una aproximación empírica", Estudios de Economía Aplicada, vol 21, 1, pp. 27-52.

MAROTO ACÍN, J.A. (1996): "Estructura financiera y crecimiento de las PYMES", Economía Industrial, 310, 4, pp. 29-40.

MATO, G. (1990): "Un análisis econométrico de la política de endeudamiento de las empresas con datos de panel", Investigaciones Económicas, vol. XIV, nº 1, pp. 6383.

MELLE HERNÁNDEZ, M. (2001): "Características diferenciales de la financiación entre las PYMES y las grandes empresas españolas", Papeles de Economía Española, no 89/90, pp. 140-141.

MENÉNDEZ REQUEJO, S. (2001): "Endeudamiento de PYMES vs grandes empresas: determinantes y relaciones estructurales", IX Foro de Finanzas, Navarra, noviembre.

MYERS, S. (1977): "Determinants of corporate borrowing", Journal of Financial Economics, 5, 2, pp. 147-175.

MYERS, S. (1984): “The capital structure puzzle”, Journal of Finance, 39, 3, pp. 575592.

MYERS, S. (2001):"Capital Structure", Journal of Economic Perspectives, vol. 15, 2, pp. 81-102.

MYERS, S. y MAJLUF, N. (1984): "Corporate financing and investment decisions when firms have information that investors do not have", Journal of Financial Economics, 13, 2, pp. 187-21.

MODIGLIANI, F. y MILLER, M. (1958): "The cost of capital, corporate finance, and the theory of investment", American Economic Review, 48,4, pp. 262-297. 
Revista Eletrônica de Ciência Administrativa (RECADM) - ISSN 1677-7387

Faculdade Cenecista de Campo Largo - Coordenação do Curso de Administração

v. 4, n. 1, maio/2005 - http://revistas.facecla.com.br/index.php/recadm/

MODIGLIANI, F. y MILLER, M. (1963): "Corporate income taxes and the cost of capital: a correction", American Economic Review, 53, 3, pp. 443-453.

OCAÑA, C.; SALAS, V. y VALLÉS, J. (1994): "Un análisis empírico de la financiación de la PYME manufacturera española", Moneda y Crédito, nº 199, pp. 57-96.

RAJAN, R. y ZINGALES, L. (1995): "What do we know about capital structure? Some evidence from international data", Journal of Finance, 50, 5, pp.1421-1460.

RITA, R. y VIDIGAL DA SILA, J. (2004): "Os determinantes da decisao de financiamento das empresas em Portugal", XIV Jornadas Hispanolusas de Gestión Científica, Febrero, Las Azores, Portugal.

SAÁ-REQUEJO, J. (1991): "Los determinantes de las decisiones sobre fuentes de financiación de las empresas españolas", Moneda y Crédito, nº 193, pp. 147-178.

SÁNCHEZ-VIDAL, J. y MARTÍN UGUEDO, J.F. (2003): "Preferencias dinámicas de financiación de las empresas españolas: nueva evidencia de la teoría de la jerarquía", XI Foro de Finanzas, Noviembre, Alicante.

SCHERER, F.M. y ROSS, D. (1990): Industrial market structure and economic performance, 3 ed. Boston: Hougthon Mifflin Company.

SHYMAN-SUNDER, L. y MYERS, S. (1999): "Testing static tradeoff against pecking order models of capital structure", Journal of Financial Economics, 51, pp. 219-244.

SOGROB-MIRA F. y LOPÉZ-GRACÍA, J. (2003): "Pecking order versus trade off: an empirical approach to the small and medium enterprise capital structure", XI Foro de Finanzas, Noviembre, Alicante.

TITMAN, S. y WESSELS, R. (1988): "The determinants of capital structure choice", Journal of Finance, 43,1,pp.1-19.

WATSON, R. y WILSON, N. (2002): "Small and medium size enterprise financing: a note on some of empirical implications of pecking order", Journal of Business Finance and Accounting, 29, 3\&4, pp. 557-578.

WARNER, J. B. (1977): "Bankruptcy costs: some evidence", Journal of Finance, 32, pp. 337-348. 